\title{
Superfluid density of two-dimensional weakly interacting boson system at zero temperature
}

\author{
HE PeiSong $^{1 *} \&$ FAN YuWei ${ }^{2}$ \\ ${ }^{1}$ School of Physics, Peking University, Beijing 100871, China; \\ ${ }^{2}$ Yuanpei College, Peking University, Beijing 100871, China
}

Received September 29, 2010; accepted December 26, 2010

\begin{abstract}
We compute the superfluid density of a two-dimensional boson system with weak two-body repulsive interactions at zero temperature using one-loop perturbation theory in the weak coupling region. The boson fields are taken to be in continuum form in real space, and the interactions are approximated by a $\delta$ function of the distance between the bosons. We find that the one-loop fluctuations slightly decrease the superfluid density of a classical level. The superfluid density is approximated by the condensate density multiplied by the mass of the boson particle.
\end{abstract}

superfluid density, one-loop fluctuations, weakly interacting

Citation: He P S, Fan Y W. Superfluid density of two-dimensional weakly interacting boson system at zero temperature. Chinese Sci Bull, 2011, 56: 1230-1233, doi 10.1007/s11434-011-4416-0

Since the remarkable experimental realization of BoseEinstein condensates (BECs) [1-3], there has been an explosion of experimental and theoretical activity devoted to the physics of dilute ultracold gases [4-6], which has led to new progress in applied physics, as well as progress in our understanding of fundamental physical knowledge. The techniques developed for creating BECs have been used in many other fields, such as the advancement of higher performance optical atomic clocks [7]. Interesting excitations have been created in ultracold gases under certain situations. Some examples of these are nonlinear matter waves [8,9], vortex-soliton modes [10] and fractionalized vortices [11].

Superfluidity is another intriguing macroscopic quantum phenomena intimately related to BECs. Superfluidity was first discovered by Kapitza in 1937 in the liquid state of Helium-4 [12]. When the temperature drops below $2.17 \mathrm{~K}$, the liquid Helium becomes superfluid, meaning that the Helium atoms flow without friction.

It is well known that there can be no long-range order in a homogeneous two-dimensional boson system with

*Corresponding author (email: heps@pku.edu.cn) short-range interactions at finite temperature according to the Mermin-Wagner-Hohenberg theorem [13-15]. Hence, the formation of a two-dimensional BEC would be impossible. However, a two-dimensional system can take on a quasilong-range topological order, forming a condensate, and become superfluid below a finite critical temperature, called the Berezinskii-Kosterlitz-Thouless (BKT) transition [16]. Accordingly, the formation of a BEC is possible after all in a two-dimensional boson system because of the possibility of an induced superfluid state with long-range order. A unique characteristic of a superfluid state is that the superfluid density $\rho_{\mathrm{s}}$ is nonzero. Therefore, to affirm the existence of a superfluid state, we need to calculate $\rho_{\mathrm{s}}$. The system that we consider is a two-dimensional homogeneous boson system with weak repulsive short-range interactions at zero temperature. We examine the effect of fluctuations in the density $\rho_{\mathrm{s}}$ of this system.

The Josephson relation [17-19] establishes a connection between the superfluid density $\rho_{\mathrm{s}}$ and the condensate density $n_{0}$, which is written as

$$
\rho_{\mathrm{s}}=-\lim _{\boldsymbol{k} \rightarrow 0} \frac{n_{0} m^{2}}{k^{2} G_{11}(\boldsymbol{k}, 0)},
$$


where $G_{11}(\boldsymbol{k}, 0)$ is the single particle Green's function with momentum $\boldsymbol{k}$ and with zero Matsubara frequency. The Josephson relation was originally derived by heuristic arguments. More recently, Holzmann and Baym [20] have derived the Josephson relation within the framework of diagrammatic perturbation theory. Their result is applicable to the twodimensional case, although it differs somewhat from eq. (1). In this paper, we use Holzmann and Baym's result to compute the superfluid density up to one-loop perturbations.

\section{The model and definition of superfluid density}

The partition function of our model can be written as a coherent state path integral over the boson field $\psi(r, \tau)$ :

$$
\begin{aligned}
\mathcal{Z}= & \int \mathcal{D} \psi \exp \left(-\frac{1}{\hbar} \int_{0}^{\hbar / k_{B} T} \mathrm{~d} \tau \mathcal{L}(\tau)\right), \\
\mathcal{L}= & \int \mathrm{d}^{2} \boldsymbol{x}\left[\hbar \psi^{*}(\boldsymbol{x}, \tau) \frac{\partial \psi(\boldsymbol{x}, \tau)}{\partial \tau}\right. \\
& \left.-\frac{\hbar^{2}}{2 m} \psi^{*}(\boldsymbol{x}, \tau) \nabla^{2} \psi(\boldsymbol{x}, \tau)-\mu|\psi(\boldsymbol{x}, \tau)|^{2}\right] \\
& +\frac{1}{2} \int \mathrm{d}^{2} \boldsymbol{x} \mathrm{d}^{2} \boldsymbol{x}^{\prime}|\psi(\boldsymbol{x}, \tau)|^{2} \lambda\left(\boldsymbol{x}-\boldsymbol{x}^{\prime}\right)\left|\psi\left(\boldsymbol{x}^{\prime}, \tau\right)\right|^{2},
\end{aligned}
$$

where $\mu$ is the chemical potential and $\lambda\left(\boldsymbol{x}-\boldsymbol{x}^{\prime}\right)$ is the interaction between two bosons at site $\boldsymbol{x}$ and $\boldsymbol{x}^{\prime}$. We consider only the case when $\lambda\left(\boldsymbol{x}-\boldsymbol{x}^{\prime}\right)=\lambda \delta\left(\boldsymbol{x}-\boldsymbol{x}^{\prime}\right) \hbar^{2} / 2 m$. In this case, the momentum cutoff of the boson field $\Lambda$ is approximately infinite. The interaction term then becomes $(1 / 2)\left(\hbar^{2} / 2 m\right) \int \mathrm{d}^{2} \boldsymbol{x}|\psi(\boldsymbol{x}, \tau)|^{2} \lambda|\psi(\boldsymbol{x}, \tau)|^{2}$. Here, $\lambda$ is dimensionless and the temperature $T$ is taken to be zero. For simplicity, during the following calculation, we set the coefficient $\hbar^{2} / 2 m$ equal to one, but we note that this coefficient is reintroduced in the final result.

Following Holzmann and Baym [20], who assumed that a condensate occurs when superfluid component exists, we consider the superfluid density. Writing the superfluid velocity as $\boldsymbol{v}_{\mathrm{s}}$, the boson field can be written as

$$
\psi\left(x, \tau ; v_{\mathrm{s}}\right)=\tilde{\psi}(\boldsymbol{x}, \tau)+\Delta_{0} \mathrm{e}^{i \frac{1}{\hbar} m v_{\mathrm{s}} \cdot x},
$$

where $\Delta_{0}=\sqrt{n_{0}}$ and $n_{0}$ is the condensate density evaluated at $v_{\mathrm{s}}=0$. The superfluid density is defined as

$$
\mathcal{F}\left(v_{\mathrm{s}} \neq 0\right)=\mathcal{F}\left(v_{\mathrm{s}}=0\right)+\frac{1}{2} \rho_{\mathrm{s}} v_{\mathrm{s}}^{2},
$$

where $\mathcal{F}$ is the free energy density of the system. The boson field $\psi\left(\boldsymbol{x}, \tau ; \boldsymbol{v}_{\mathrm{s}}\right)$ in eq. (3) can be rewritten in the following form:

$$
\psi\left(\boldsymbol{x}, \tau ; \boldsymbol{v}_{\mathrm{s}}\right)=\psi\left(\boldsymbol{x}, \tau ; \boldsymbol{v}_{\mathrm{s}}=0\right)+\delta \psi(\boldsymbol{x})
$$

with

$$
\delta \psi(\boldsymbol{x})=\Delta_{0}\left(\mathrm{e}^{i \frac{1}{\hbar} m v_{\mathrm{s}} \cdot \boldsymbol{x}}-1\right),
$$

where $\delta \psi(\boldsymbol{x})$ is defined as the variation of the condensate density due to the existence of a finite superfluid velocity. Putting these expressions into the partition function for the system (2) and including the definition of superfluid density (4), we obtain the superfluid density

$$
\begin{aligned}
\rho_{\mathrm{S}}= & \frac{m^{2} \Delta_{0}^{2}}{2 \hbar^{2}} \lim _{\boldsymbol{k} \rightarrow 0}\left(\hat{\boldsymbol{v}}_{\mathrm{S}} \cdot \nabla_{\boldsymbol{k}}\right)^{2}\left[\mathcal{G}_{11}^{-1}(\boldsymbol{k}, \omega=0)-\mathcal{G}_{12}^{-1}(\boldsymbol{k}, \omega=0)\right. \\
& \left.-\mathcal{G}_{21}^{-1}(\boldsymbol{k}, \omega=0)+\mathcal{G}_{22}^{-1}(\boldsymbol{k}, \omega=0)\right] .
\end{aligned}
$$

Here $\mathcal{G}$ is the $2 \times 2$ matrix defined as the propagator of the combined fields $\delta \Psi^{*}(\boldsymbol{x}, \tau)=\left(\delta \psi^{*}(\boldsymbol{x}, \tau), \delta \psi(\boldsymbol{x}, \tau)\right)$. Because $\delta \psi(\boldsymbol{x})$ has no $\tau$ dependence, $\mathcal{G}(\boldsymbol{k}, \omega)$ in eq. (7) yields $\omega=0$.

For our system, we can write eq. (5) as

$$
\psi\left(\boldsymbol{x}, \tau ; \boldsymbol{v}_{\mathrm{s}}\right)=\Delta_{0}+\tilde{\psi}\left(\boldsymbol{x}, \tau ; \boldsymbol{v}_{\mathrm{s}}=0\right)+\delta \psi(\boldsymbol{x}) .
$$

From eq. (8) we see that the Green's functions for $\delta \psi(\boldsymbol{x})$ are the same as those for $\tilde{\psi}\left(\boldsymbol{x}, \tau ; \boldsymbol{v}_{\mathrm{s}}=0\right)$, except that $\omega$ is not zero in the latter Green's function.

Using the relation in eq. (7) for the equilibrium case with $\boldsymbol{v}_{\mathrm{s}}=0$, that is, omitting $\delta \psi(\boldsymbol{x})$ we find $\psi\left(\boldsymbol{x}, \tau ; \boldsymbol{v}_{\mathrm{s}}=0\right)$ instead of $\psi\left(\boldsymbol{x}, \tau ; \boldsymbol{v}_{\mathrm{s}}\right)$ in the Lagrangian. We note that we have assumed the $\hat{k} x$ direction in the derivation for eq. (7).

\section{One-loop corrections for superfluid density}

For convenience, we write the equilibrium field as

$$
\psi(\boldsymbol{x}, \tau)=\sqrt{\frac{1}{2}}(v+\chi(\boldsymbol{x}, \tau)) .
$$

Here, we take $v$ to be real because of the $\mathrm{U}(1)$ symmetry of the system, where $v$ equals $\sqrt{2} \Delta_{0}$, compared to Holzmann and Baym's paper. The complex boson field can be written as $\chi=\chi_{1}+i \chi_{2}$, where $\chi_{1}$ and $\chi_{2}$ are the real and imaginary parts of $\chi$ respectively.

Writing the Lagrangian eq. (2) in terms of $\chi_{1}$ and $\chi_{2}$, we obtain the following expression for the partition function:

$$
\mathcal{Z}=\mathrm{e}^{-\beta V\left(-\frac{\mu}{2} v^{2}+\frac{\lambda}{8} v^{4}\right)} \int D \chi_{1} D \chi_{2} \mathrm{e}^{-\int \frac{\mathrm{d} \omega}{2 \pi} \int \frac{\mathrm{d}^{2} k}{4 \pi^{2}}\left[\frac{1}{2} \chi_{a} \hat{M}_{a b} \chi_{b}+\mathcal{L}_{\mathrm{int}}\right]},
$$

$M_{a b}=\left(\begin{array}{cc}\epsilon_{k}-\mu+3 \lambda v^{2} / 2 & -\omega \\ \omega & \epsilon_{k}-\mu+\lambda v^{2} / 2\end{array}\right)$,

$\mathcal{L}_{\text {int }}=\frac{\lambda}{8}(\chi \cdot \chi)^{2}+\frac{1}{2} \lambda v \chi_{1}(\chi \cdot \chi)$,

where $a, b=1,2$, respectively.

In the classical limit, the free energy density is

$$
\frac{1}{V} \Omega_{\mathrm{cl}}(\mu, v)=-\frac{\mu}{2} v^{2}+\frac{\lambda}{8} v^{4} .
$$

The minimum of $\Omega_{\mathrm{cl}}$ with respect to $v^{2}$ if found when $\partial \Omega_{\mathrm{cl}} / \partial v^{2}=0$, which leads to $v_{\mathrm{cl}}^{2}=2 \mu / \lambda$. The condensate density in the classical limit is

$$
n_{\mathrm{cl}}=\frac{\mu}{\lambda} .
$$


Because $\lambda>0$, for $v$ to be nonzero, the chemical potential $\mu$ must be positive. The phase transition point is located at $\mu=0$. When $\mu$ is negative, there is no macroscopic condensate of particles in the ground state.

Using this relation, we obtain the free propagator of $\chi$ in the basis $\left(\chi_{1}, \chi_{2}\right)$ to be

$$
G^{(0)}(\boldsymbol{k}, i \omega)=\frac{1}{\omega^{2}+\epsilon_{k}\left(\epsilon_{k}+2 \mu\right)}\left(\begin{array}{cc}
\epsilon_{k} & \omega \\
-\omega & \epsilon_{k}+2 \mu
\end{array}\right)
$$

where $\epsilon_{k}=\hbar^{2} k^{2} / 2 m$. The dispersion relation becomes

$$
\omega_{k}=\sqrt{\epsilon_{k}\left(\epsilon_{k}+2 \mu\right)}=\frac{\hbar^{2}}{2 m} k \sqrt{k^{2}+\frac{4 m \mu}{\hbar^{2}}} .
$$

For small $k$, the dispersion relation is gapless and linear. According to [21], this appearance of "phonon"-like dispersion has one-to-one correspondence to superfluidity. So we show that the assumption in Holzmann and Baym's work for the derivation of eq. (7) is fulfilled.

In the one-loop approximation,

$G^{-1}(\boldsymbol{k}, i \omega)=\left(\begin{array}{cc}\frac{\hbar^{2} k^{2}}{2 m}-\mu+\frac{3}{2} \lambda v^{2}+\Pi_{11} & -\omega+\Pi_{12} \\ \omega+\Pi_{21} & \frac{\hbar^{2} k^{2}}{2 m}-\mu+\frac{1}{2} \lambda v^{2}+\Pi_{22}\end{array}\right)$.

In the new field basis, the superfluid density in eq. (7) can be written as

$$
\rho_{\mathrm{s}}=\left.\frac{n_{0} m^{2}}{\hbar^{2}} \frac{\partial^{2}}{\partial k_{x}^{2}}\left[\epsilon_{k}+\Pi_{22}(\boldsymbol{k}, 0)+i \Pi_{12}(\boldsymbol{k}, 0)\right]\right|_{\boldsymbol{k} \rightarrow 0},
$$

where

$$
\begin{aligned}
\Pi_{22}(\boldsymbol{k}, i \omega)= & \frac{\hbar^{2}}{2 m} \int \frac{\mathrm{d}^{2} \boldsymbol{q} \mathrm{d} v}{(2 \pi)^{3}}\left[3\left(-\frac{\lambda}{2}\right) G_{22}^{(0)}(\boldsymbol{q}, i v)\right. \\
& +\left(-\frac{\lambda}{2}\right) G_{11}^{(0)}(\boldsymbol{q}, i v) \\
& +4\left(-\frac{\lambda}{2} v\right)^{2} G_{11}^{(0)}(\boldsymbol{q}, i v) G_{22}^{(0)}(\boldsymbol{k}+\boldsymbol{q}, i \omega+i v) \\
& \left.+4\left(-\frac{\lambda}{2} v\right)^{2} G_{12}^{(0)}(\boldsymbol{q}, i v) G_{21}^{(0)}(\boldsymbol{k}+\boldsymbol{q}, i \omega+i v)\right], \\
\Pi_{12}(\boldsymbol{k}, i \omega)= & \frac{\hbar^{2}}{2 m} \int \frac{\mathrm{d}^{2} \boldsymbol{q} \mathrm{d} v}{(2 \pi)^{3}}\left[4\left(-\frac{\lambda}{2} v\right)^{2} G_{12}^{(0)}(\boldsymbol{q}, i v)\right. \\
& \times G_{22}^{(0)}(\boldsymbol{k}+\boldsymbol{q}, i \omega+i v) \\
& \left.+12\left(-\frac{\lambda}{2} v\right)^{2} G_{11}^{(0)}(\boldsymbol{q}, i v) G_{12}^{(0)}(\boldsymbol{k}+\boldsymbol{q}, i \omega+i v)\right](19)
\end{aligned}
$$

are depicted in Figure 1.

Differentiating $\Pi_{22}$, we obtain

$$
\left.\frac{\partial^{2} \Pi_{22}(k, 0)}{\partial k_{x}^{2}}\right|_{k=0}=-\frac{\hbar^{2}}{2 m} \frac{\left(-\frac{\lambda}{2} v\right)^{2}}{6 \pi \mu} \frac{1}{\left(1+\frac{2 m}{\hbar^{2}} \frac{2 \mu}{\Lambda^{2}}\right)^{\frac{3}{2}}} \simeq-\frac{\hbar^{2}}{2 m} \frac{\lambda}{12 \pi} .
$$

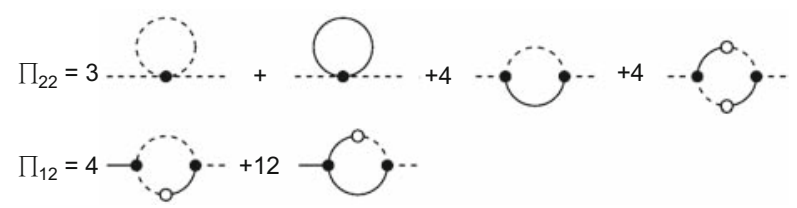

Figure 1 One-loop corrections of self energy for the propagators $G(\boldsymbol{k}, i \omega)$.

Furthermore, the $\Pi_{12}$ term yields the zero contributions obtained when integrating over odd functions.

Finally, from eq. (18), we arrive at the main result of this paper, which portrays the superfluid density in the following form:

$$
\rho_{s}=\frac{m \mu}{\lambda} \frac{2 m}{\hbar^{2}}\left[1-\frac{\lambda}{24 \pi}\right] .
$$

It is clear from this result that one-loop fluctuations decrease the superfluid density somewhat. The correction is a small effect compared to the classical approximation. The superfluid density is approximated by the condensate density multiplied by the mass of the boson particle. Our result that the repulsive interactions reduce the density of particles in the condensate has been similarly shown for the case of a weakly interacting Fermi gas [22].

\section{Conclusions}

In this paper, using the result obtained by Holzmann and Baym, we have calculated the superfluid density of a twodimensional weakly interacting boson system at zero temperature up to the order of one-loop perturbations. We have found that the density fluctuations due to one-loop perturbations decrease the superfluid density slightly compared to the classical approximation. Finally, we have shown that the superfluid density can be approximated by the condensate density multiplied by the mass of bosons.

1 Anderson M H, Ensher J R, Matthews M R, et al. Observation of BoseEinstein condensation in a dilute atomic vapor. Science, 1995, 269: 198-201

2 Bradley C C, Sackett C A, Tollett J J, et al. Evidence of Bose-Einstein condensation in an atomic gas with attractive interactions. Phys Rev Lett, 1995, 75: 1687-1690

3 Davis K B, Mewes M O, Andrews M R, et al. Bose-Einstein condensation in a gas of sodium atoms. Phys Rev Lett, 1995, 75: 3969-3973

4 Xu Z, Zhou S Y, Zhou S Y, et al. Production and measurement of BoseEinstein condensate of ${ }^{87} \mathrm{Rb}$ atomic gas. Sci China Ser G-Phys Mech Astron, 2008, 51: 22-28

5 Shao T L. Dynamic structure factor of a Bose-Hubbard gas. Chinese Sci Bull, 2008, 53: 2432-2437

6 Duan Z L, Steel M J, Xu A T, et al. Effective mass approach for a BoseEinstein condensate in an optical lattice. Chinese Sci Bull, 2009, 54: 4182-4196

7 Chen J B. Active optical clock. Chinese Sci Bull, 2009, 54: 348-352

8 Wang D S, Hu X H, Hu J P, et al. Quantized quasi-two-dimensional Bose-Einstein condensates with spatially modulated nonlinearity. Phys Rev A, 2010, 81: 025604-025607

9 Zhu S H, Zhang J, Li X G. Limiting profile of blow-up solutions for the Gross-Pitaevskii equation. Sci China Ser A-Math, 2009, 52: 1017-1030 
10 Wu L, Li L, Zhang J F, et al. Exact solutions of the Gross-Pitaevskii equation for stable vortex modes in two-dimensional Bose-Einstein condensates. Phys Rev A, 2010, 81: 061805-061808

11 Ji A C, Liu W M, Song J L, et al. Dynamical creation of fractionalized vortices and vortex lattices. Phys Rev Lett, 2008, 101: 010402-010405

12 Lifshitz E M, Pitaevskii L P. Statistical Physics. Oxford: Pergamon Press, 1980

13 Mermin N D, Wagner H. Absence of ferromagnetism or antiferromagnetism in one- or two-dimensional isotropic Heisenberg models. Phys Rev Lett, 1966, 17: 1133-1136

14 Wagner H. Long-wavelength excitations and the Goldstone theorem in many-particle systems with broken symmetries. Z Phys, 1966, 195: 273-299

15 Hohenberg P C. Existence of long-range order in one and two dimensions. Phys Rev, 1967, 158: 383-386

16 Kosterlitz M, Thouless D. Order, metastability and phase transitions in two-dimensional systems. J Phys C, 1973, 6: 1181-1203

17 Josephson B D. Relation between the superfluid density and order parameter for superfluid He near $T_{c}$. Phys Lett, 1966, 21: 608-609

18 Baym G. Mathematical Methods in Solid State and Superfluid Theory. Clark R C, Derrick G H, eds. Edinburgh: Oliver and Boyd, 1969. 121

19 Holzmann M, Baym G. Condensate density and superfluid mass density of a dilute Bose-Einstein condensate near the condensation transition. Phys Rev Lett, 2003, 90: 040402-040405

20 Holzmann M, Baym G. Condensate superfluidity and infrared structure of the single-particle Greens function: The Josephson relation. Phys Rev B, 2007, 76: 092502-092504

21 Nagaosa N. Quantum Field Theory in Condensed Matter Physics. Heidelberg: Springer, 1999

22 Men F D, Liu H, Zhu H Y. Unified properties of a weakly interacting Fermi gas in a weak magnetic field. Sci China Ser G-Phys Mech Astron, 2008, 51: 1072-1078

Open Access This article is distributed under the terms of the Creative Commons Attribution License which permits any use, distribution, and reproduction in any medium, provided the original author(s) and source are credited. 